\title{
Grado de adherencia terapéutica a los fármacos del metabolismo óseo-mineral: ¿toman nuestros pacientes la medicación prescrita?
}

\author{
María Nieves Cazorla Santana, Dora Rodríguez Díaz
}

Enfermeras. Centro de Hemodiálisis Avericum, Gran Canaria

\section{Resumen}

Los fármacos del metabolismo óseo-mineral tienen gran importancia el tratamiento de los pacientes de hemodiálisis, pero desconocemos las preferencias, y el grado de conocimiento de estos fármacos. Los objetivos del estudio fueron conocer el grado de adherencia terapéutica a los fármacos del metabolismo oseo-mineral, e identificar los factores más relevantes que influyen en la adherencia a este tipo de tratamiento.

Pacientes y métodos: Se realizó un estudio transversal en un Centro de hemodiálisis, incluyendo 106 pacientes. Utilizamos el cuestionario simplificado de adherencia a la medicación $(S M A Q)^{1}$ y un cuestionario específico sobre cumplimiento y preferencias, para conocer el grado de adherencia.

Resultados: El $37,7 \%$ de los pacientes fue incumplidor según el cuestionario SMAQ. De ellos, el $34 \%$ no tomaba la medicación a la misma hora $(P<0.000)$, el $5,7 \%$ incumplía el tratamiento los fines de semana $(P<0.010)$, el $13,2 \%$ dejan de tomar la medicación cuando se encuentran mal $(P<0.010) ; y$ en la última semana, el $18 \%$ reconocía que olvidó el tratamiento ldía $(P=0.001)$ y entre $2-3$ días $(12,5 \%)$. El $52 \%$ no tiene preferencias por ningún quelante del fósforo y el $60.4 \%$ conocía la utilidad de los mismos. La principal razón de desagrado fue el aumento del consumo de agua

\section{Correspondencia: \\ María Nieves Cazorla Santana \\ C/ Ignacio Ellacuría 20 \\ 35200, Telde, Las Palmas de Gran Canaria \\ E-mail: marinievescazorlasantana@gmail.com}

( $16 \%$ ), seguido del tamaño del comprimido (15\%) y el carbonato de lantano es el fármaco que menos les gusta. Finalmente, el 91\% reconoce recibir información dietética.

Conclusiones: Como conclusión de nuestro estudio, el $37,7 \%$ de los pacientes en programa de hemodiálisis de nuestro centro, fue no adherente al tratamiento médico prescrito, según las respuestas obtenidas en el cuestionario $S M A Q$, y que el grado de conocimiento, información y/o compresión sobre el tratamiento médico-dietético prescrito a los pacientes son factores muy importantes para mejorar el grado de cumplimiento, por lo que la implicación activa el personal de enfermería constituye una herramienta clave para conseguir el objetivo terapéutico.

PALABRAS CLAVE:

- FÁRMACOS DEL METABOLISMO ÓSEO-MINERAL

- ADHERENCIA TERAPÉUTICA

- PREFERENCIAS

- CUMPLIMIENTO TERAPÉUTICO

- HEMODIÁLISIS

Degree of therapeutic adherence to bone mineral metabolism drugs: Do our patients take the prescribed medication?

\section{Abstract}

Bone mineral metabolism drugs are of great importance in treating haemodialysis patients, but we are unaware of their preferences and degree of knowl- 
edge of these drugs. The aims of the study were to find out the degree of therapeutic adherence to bone mineral metabolism drugs and to identify the most relevant factors that influence adherence to this type of treatment.

Patients and methods: A transversal study was carried out at a haemodialysis centre, including 106 patients. We used the simplified medication adherence questionnaire $(S M A Q)^{1}$ and a specific questionnaire on compliance and preferences, in order to find out the degree of adherence.

Results: $37.7 \%$ of the patients were non-compliant, according to the SMAQ. Of these, $34 \%$ did not take the medication at the same time $(P<0.000), 5.7 \%$ did not comply with the treatment at weekends $(P<0.010), 13.2 \%$ stop taking the medication when they feel unwell $(P<0.010)$; and in the last week, $18 \%$ acknowledged that they forgot the treatment 1 day $(P=0.001)$ and between $2-3$ days $(12,5 \%)$. $52 \%$ did not have preferences for any phosphorus chelant and $60.4 \%$ knew about their usefulness. The main reason for dislike was the increase in water consumption ( $16 \%$ ), followed by the size of the tablet (15\%) and lanthanum carbonate was the drug they liked least. Finally, 91\% acknowledged that they had received dietary information.

Conclusions: As conclusions of our study, $37.7 \%$ of the patients on the haemodialysis programme at our centre did not adhere to the prescribed treatment, according to the answers obtained in the SMAQ, and the degree of knowledge, information and/or understanding of the medical and dietary treatment prescribed to patients are very important factors in improving the degree of compliance, and therefore the active involvement of nursing staff is a key tool in order to achieve the therapeutic goal.

\section{KEY WORDS:}

- BONE MINERAL METABOLISM DRUGS

- THERAPEUTIC ADHERENCE - PREFERENCES. THERAPEUTIC COMPLIANCE - HAEMODIALYSIS

\section{Introducción}

La adherencia terapéutica es un fenómeno complejo, que está condicionado por múltiples factores de diversa naturaleza, sin embargo, es un comportamiento humano, modulado por componentes subjetivos y en esta dimensión debe ser esencialmente comprendido, abordado e investigado ${ }^{2}$. El incumplimiento o falta de adherencia terapéutica constituye un importante problema de salud pública. Estudios previos han descrito que la falta de adherencia al tratamiento oscila, según la patología, entre el $30 \%$ y el $60 \%$ de los pacientes, y en al menos en la mitad de los pacientes, los beneficios potenciales del tratamiento prescrito se ven mermados a causa de este incumplimiento $3,4,5,6,7,8$. Una incorrecta adherencia farmacológica incluye aspectos como la omisión de tomas, reducción de la dosis prescrita, no respetar los intervalos o frecuencias de administración correctas, no ajustarse a los requerimientos de administración en relación con las comidas $u$ otras circunstancias.Concretamente, en pacientes con enfermedad renal crónica en tratamiento sustitutivo de la función renal mediante diálisis (Estadio 5-D), tanto la adherencia al tratamiento dialítico, así como la adherencia al tratamiento farmacológico tiene un impacto directo sobre supervivencia del paciente. En estos pacientes el mal cumplimiento puede ser producto por la falta de información y/o comprensión sobre el tratamiento farmacológico, por la cronicidad de la enfermedad o la polimedicación.

En la actualidad la mayoría de los pacientes que precisan tratamiento sustitutivo de la función renal presentan una gran comorbilidad (Enfermedad cardiovascular, diabetes mellitus, ateromatosis, hipertensión arterial, etc.) siendo necesario ser tratados con un elevado número de fármacos, lo que puede provocar en ocasiones un abandono del tratamiento con la consiguiente disminución de la adherencia farmacológica. En este sentido, la falta de cumplimiento terapéutico a los fármacos del metabolismo óseo-mineral ( MOM) dificulta alcanzar los objetivos terapéuticos. Hoy por hoy, el control del fósforo y del hiperparatiroidismo constituye un gran problema en las unidades de diálisis donde los objetivos son cada vez más ambiciosos y pueden diferir mucho de la realidad $^{3}$

Es bien sabido, que los fármacos del MOM presentan ciertas características que lo hacen diferentes al resto de fármacos y eso en gran medida puede que dificulte la adherencia al tratamiento ${ }^{3}$, por ello, los objetivos del estudio fueron conocer el grado de adherencia terapéutica a los fármacos del metabolismo oseo-mineral, e 
identificar los factores más relevantes que influyen en la adherencia a este tipo de tratamiento.

\section{Metodología}

Se llevó a cabo un estudio transversal en centro satélite de hemodiálisis, durante un periodo de cuatro meses, donde fueron incluidos 106 pacientes.

Los criterios de inclusión fueron los pacientes con edad superior a 18 años, con un tiempo de permanencia superior a tres meses en programa de hemodiálisis y que tomaban habitualmente algún fármaco del MOM, durante un tiempo mínimo al menos, de tres meses.

Se consideraron criterios de exclusión, las condiciones psíquicas inadecuadas (considerándose únicamente el deterioro intelectual severo que impida la realización de los cuestionarios), los pacientes éxitus y traslados con pérdida de seguimiento, los pacientes que no completaron los 2 cuestionarios y las negativas de los pacientes a realizar el estudio.

Para hacer la valoración del grado de adhesión terapéutica se utilizó el cuestionario genérico de cumplimiento del tratamiento (SMAQ) cuestionario validado en la población española con SIDA ${ }^{2}$. Este cuestionario ha sido recientemente validado en pacientes con trasplanterenal ${ }^{4} y$ previamente utilizado en pacientes en programa de hemodiálisis crónica ${ }^{3}$. El cuestionario SMAQ consiste en solicitar al paciente que conteste unas preguntas previamente definidas y en función de sus respuestas, poder valorar el grado de adherencia (tabla 1). Se considera al paciente adherente, cuando tiene un cumplimiento terapéutico igual o superior al $95 \%$.

Además, utilizamos un cuestionario específico sobre cumplimiento y preferencias de los pacientes por los fármacos del MOM (anexo 1).

En este estudio, se investigaron los posibles factores que pudieran estar relacionados con la no adherencia al tratamiento, para lo cual, la variable dependiente fue la adherencia terapéutica (olvida o no tomar la medicación prescrita) en los pacientes en programa de hemodiálisis al tratamiento farmacológico y como variables independientes, aquellas que hacen referencia a aspectos sociodemográficos, médico-clínicos y a parámetros específicos de la enfermedad renal (edad, sexo, tiempo en HD, nivel socioeconómico, cultural y etiología de la Enfermedad Renal Crónica).

\section{- Análisis estadístico}

Los datos obtenidos han sido objeto de codificación y tratados informáticamente, mediante el programa estadístico SPSS versión 17 para Windows ${ }^{\circledR}$. (Statistical Packagefor Social Science) (Chicago, IL, USA). Se realizó un test de Kolmogorov-Smirnov para verificar que las variables seguían una distribución normal. Los datos se expresaron como media y desviación estándar o mediana y percentiles.

Para la comparación de proporciones hemos utilizado el Chi2 y la comparación de medias se realizó con la $t$ de student. Se consideró un valor estadísticamente significativo cuando $\mathrm{P}<0.05$.

A todos los pacientes, se les solicitó el consentimiento informado para ser incluidos en el estudio. Una vez obtenidas las autorizaciones, los pacientes han recibido una copia del consentimiento informado el cual les ha sido leído por el encuestador.

Finalmente, el estudio se llevó a cabo según los principios de la Declaración de Helsinki.

\section{Resultados}

De 191 pacientes prevalentes, 128 tomaban algún fármaco del MOM; 19 de ellos, no estaban capacitados para completar el cuestionario; 1 negativa a realizar el estudio y 2 fueron traslados con pérdida de seguimiento, por lo que fueron excluidos.

El $71,7 \%$ de los pacientes eran varones, con una edad media de $61 \pm 13$ años, y un tiempo medio en hemodiálisis de 52,6 \pm 57 meses. En cuanto a la enfermedad de base hay una mayor prevalencia de Nefropatía Diabética, con un $47,2 \%$, seguida de Nefropatía isquémica $17 \%$. Destacar el alto porcentaje de pacientes con HTA $(90,6 \%)$ y con ECV $(38,7 \%)$ (tabla 2$)$. La mayoría de los pacientes eran pensionistas $69,8 \%$, con un nivel de estudios bajo (primarios $59,4 \%$ y el $24,5 \%$ no tenían estudios). Sólo un $12,3 \%$ de nuestros pacientes viven sólo y al 46,2\% le supervisan la medicación, bien su pareja u otro familiar.

- Cuestionario de cumplimiento SMAQ (tablal):

Según el cuestionario de cumplimiento SMAQ, el 37,7 de los pacientes fue incumplidor y de ellos el $34 \%$ no tomaba la medicación a la misma hora $(P<0.000)$, el nivel de incumplimiento durante los fines de semana 
fue de un $5,7 \%(P<0.010)$, cuando se encuentran mal, fue de un $13,2 \%(P<0.010)$ y el $18 \%$ reconocía que olvidó el tratamiento ldía $(P=0.001)$ y entre 2 -3días (12,5\%).

\begin{tabular}{|c|c|c|}
\hline $\begin{array}{l}\text { 1. Alguna vez, ¿olvida tomar la medica- } \\
\text { ción? }\end{array}$ & Si & no \\
\hline 2. ¿Toma los fármacos a la misma hora? & $\mathrm{Si}$ & no \\
\hline $\begin{array}{l}\text { 3. ¿Cuándo se encuentra mal deja de } \\
\text { tomar la medicación? }\end{array}$ & $\mathrm{Si}$ & no \\
\hline $\begin{array}{l}\text { 4. ¿Olvida tomar la medicación durante } \\
\text { el fin de semana? }\end{array}$ & $\mathrm{Si}$ & no \\
\hline $\begin{array}{l}\text { 5. En la última semana, ¿cuántas veces } \\
\text { se le olvidó? }\end{array}$ & $\begin{array}{l}\text { A. Ningún } \\
\text { día }\end{array}$ & B. 1 día \\
\hline $\begin{array}{c}\text { C. de 2-3 } \\
\text { días }\end{array}$ & $\begin{array}{l}\text { D. } 4-5 \\
\text { días }\end{array}$ & $\begin{array}{r}\text { E. }>5 \\
\text { días }\end{array}$ \\
\hline
\end{tabular}

Tabla 1. Cuestionario de cumplimiento SMAQ

- Cuestionario específico sobre cumplimiento y preferencias:

El $52 \%$ de los pacientes no tienen preferencias por los quelantes del fósforo y el fármaco que menos les gusta es el carbonato de lantano (27\%) seguido de sevelamer, acetato cálcico y carbonato cálcico (4\%). (Gráfico 1)

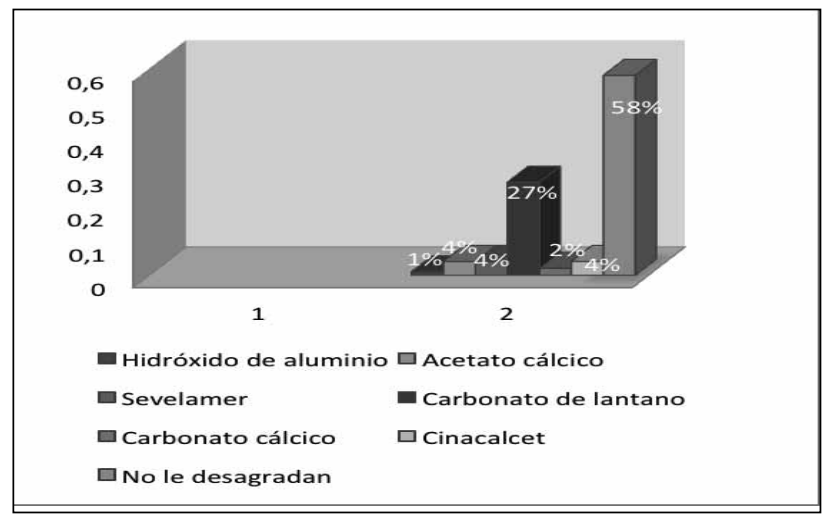

Gráfico 1. Preferencias

Las razones de desagrado fueron aumento del consumo de agua (16\%), el gran tamaño del comprimido (15\%), intolerancia gástrica (10\%), necesidad de tomar muchos comprimidos y mal sabor del fármaco (5\%). (Gráfico 2)

Al analizar el conocimiento de los pacientes respecto a la utilidad de estos fármacos, un $60.4 \%$ conocía que los quelantes sirven para controlar el fósforo; el $50,9 \%$ de la población estudiada piensa que la toma de éstos fármacos son importantes para el control

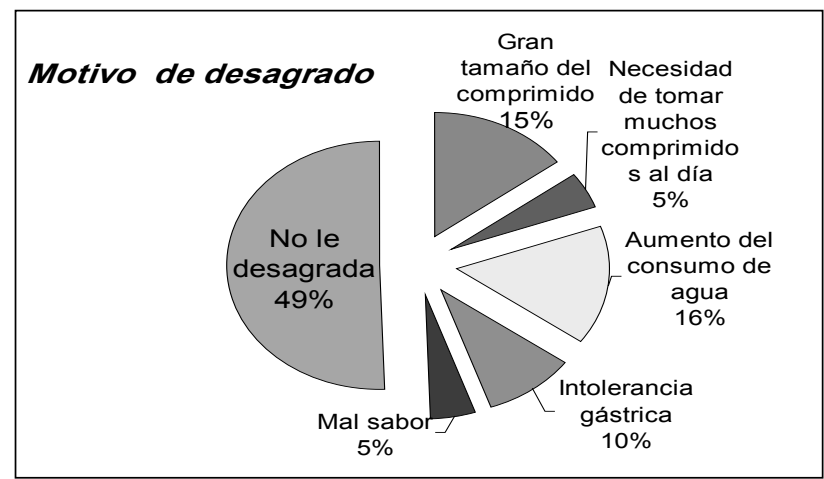

Gráfico 2. Motivo de desagrado

\begin{tabular}{ll}
\hline Edad & $61 \pm 13$ años \\
\hline Sexo & \\
\hline Mujeres & $29 \%$ \\
\hline Hombres & $71 \%$ \\
\hline Tiempo en H.D (meses) & $52.6 \pm 57$ meses \\
\hline *Enfermedad de base & \\
\hline N.Diabética & $44.3 \%$ \\
\hline NAE/Isq & $17 \%$ \\
\hline PQR & $11.3 \%$ \\
\hline No Filiada & $9.4 \%$ \\
\hline GNC & $4.7 \%$ \\
\hline Otras & $13.2 \%$ \\
\hline **ECV & $38.7 \%$ \\
\hline Hipertensión arterial & $90.6 \%$ \\
\hline Diabetes mellitus & $52.9 \%$ \\
\hline
\end{tabular}

Tabla 2. Características basales

* Enfermedad de base:

N.Diabética: Nefropatía diabética NAE/Isq.:

Nefroangioesclerosis

$P Q R$ : Poliquistosis renal

GNC: Glomerulonefritis crónica.

**ECV: Cardiopatía isquémica, Accidente cerebro vascular, Insuficiencia cardiaca congestiva y Enfermedad arterial periférica.

del fósforo; un $28,3 \%$ desconoce su importancia y el $13,2 \%$ "porque lo dice el médico" (tabla 3 ) y el $9,4 \%$ de la población que tomaba cinacalcet (35\% de los encuestados) sabía que este fármaco disminuye la secreción de PTH.

Finalmente, el $91 \%$ reconoce haber recibido la información dietética necesaria; un $76,4 \%$ afirma seguirla. 


\begin{tabular}{|l|c|c|}
\hline & ¿Para qué sirven? & $\begin{array}{c}\text { ¿Porqué son im- } \\
\text { portantes? }\end{array}$ \\
\hline $\begin{array}{l}\text { Para el control del } \\
\text { fósforo }\end{array}$ & $60.4 \%$ & $50.9 \%$ \\
\hline Para el hueso & $2 \%$ & $4.7 \%$ \\
\hline Para el corazón & $0.9 \%$ & $2.8 \%$ \\
\hline No sabe & $34 \%$ & $25.5 \%$ \\
\hline No contesta & $2.8 \%$ & $2.8 \%$ \\
\hline Porque me lo dice el & & $13.2 \%$ \\
\hline médico
\end{tabular}

Tabla 3. Importancia y utilidad de los fármacos del metabolismo óseomineral

1. ¿Vive usted sólo?
a. Si
b. No

2. ¿Tiene usted alguna persona que supervise la medicación que toma?
a. Si
b. No

3. ¿Cuándo sale de casa ¿Se toma la medicación ?
a. Si
b. No

4. De los medicamentos que se nombran a continuación ¿cuál es el que menos le gusta?
a. Acetato cálcico (Royen)
b. Carbonato cálcico (Natecal,Caosina)
c. Carbonato de lantano (Fosrenol)
d. Hidróxido de aluminio (Pepsamar)
e. Sevelamer (Renagel)
f. Cinacalcet (Mimpara)
g. No me desagrada ninguno de los probados

5. En el caso de desagradarle alguno de estos fármacos, indique la razón:
a. Gran tamaño del comprimido
b. Necesidad de tomar muchos comprimidos al día
c. Aumento del consumo de agua
d. Intolerancia gástrica
e. Mal sabor

6. ¿Usted sabe para qué sirve la medicación prescrita?
a. Para el control del fósforo
b. Para el hueso
c. Para el corazón
d. No sabe
e. No contesta

7. ¿Sabe usted porqué es importante la toma de estos medicamentos?
a. Para el control del fósforo
b. Para el hueso
c. Para el corazón
d. No sabe
e. No contesta
f. Porque me lo dice el médico

8. Además de tomar la medicación prescrita, ¿usted sigue las indicaciones dietéticas?
a. Si
b. No

9. ¿Recibe usted la información necesaria para seguir la dieta adecuada?
a. Si
b. No

10. ¿Toma usted Cinacalcet (Mimpara)?
a. Si
b. No

11. En el caso de tomar Cinacalcet (Mimpara) ¿Sabe usted para que sirve?
a. Para disminuir la PTH
b. Para disminuir el fósforo
c. Para el corazón
d. No sabe
e. No contesta

Anexo 1: cuestionario específico

\section{Discusión}

El cumplimiento del tratamiento en la enfermedad renal crónica (ERC) en sus diferentes aspectos es fundamental, y de ella dependen una gran parte de los resultados de los mismos. Nuestro estudio muestra una falta de cumplimiento del tratamiento con los fármacos prescritos en pacientes de hemodiálisis, que alcanza el $37,7 \%$, según las respuestas obtenidas en el cuestionario SMAQ.

Existen pocos estudios que traten este tema específicamente y los datos publicados varían en función de los publicaciones donde la falta de cumplimiento al tratamiento farmacológico $0^{6}$ oscila entre un 22 y un $74 \%$, con una mediana del $50 \% 3$.

Es bien sabido, que los niveles más bajos de cumplimiento se observan en pacientes crónicos y en quienes el tratamiento exige un cambio en su estilo de vida y los 
pacientes con ERC pertenecerían a este grupo de pacientes.

En general, múltiples factores se han relacionado con el incumplimiento del tratamiento. En principio, no se han encontrado relaciones claras y constantes entre el cumplimiento y las variables sociodemográficas o las características de la población, excepto en el caso de la edad donde los pacientes jóvenes tienen mayor probabilidad de ser incumplidores, ya que la interferencia con los hábitos de vida, ya sea durante el horario de trabajo 0 en ciertos momentos englobados en el contexto de la vida social del paciente, puede motivar que algunos de ellos dejen de tomar la medicación o que lo hagan en un horario incorrecto. En un estudio realizado en el $2007^{9}$ los resultados demostraron que los pacientes no cumplidores correspondieron al $49.5 \%$ de la población y no se encontró relación estadística significativa entre el grado de cumplimiento terapéutico y el resto de variables, excepto entre la técnica de diálisis utilizada y el porcentaje de cumplimiento, donde los pacientes de diálisis peritoneal fueron mejores cumplidores, así como la relación entre el nivel intelectual y el cumplimiento terapéutico, donde los pacientes que no tienen ningún tipo de estudios son menos cumplidores del régimen terapéutico. Sin embargo, en nuestro estudio no hemos encontrado una relación estadística significativa entre las distintas variables (edad, sexo, nivel cultural, estado civil o causa de entrada en diálisis) con el incumplimiento terapéutico.

Otro factor importante que influye en la falta de seguimiento del tratamiento es la complejidad y las exigencias del régimen de tratamiento, por ejemplo, el número de dosis diarias se ha relacionado de forma inversa con el cumplimiento $0^{10}$ y esto, a su vez, se asocia con una peor calidad de vida de los pacientes ${ }^{6}$.

Por otra parte, al analizar el grado de adherencia en nuestra población, donde un conocimiento adecuado de la acción de los fármacos puede influir favorablemente en el cumplimiento terapéutico, observamos que existe un gran desconocimiento sobre la importancia de los fármacos del metabolismo óseo-mineral (MOM). De los pacientes estudiados $(28,3 \%)$ desconocían la magnitud de su importancia. Transmitir adecuadamente la importancia de estos fármacos es fundamental y puede contribuir a aumentar el cumplimiento del tratamiento11,12.

Por otro lado, conocer las preferencias de los pacientes acerca de los fármacos del MOM prescritos puede ser otro factor importante a la hora de conseguir un adecuado seguimiento del tratamiento, ya que, basándose en el conocimiento de las preferencias de los pacientes se podrían prescribir fármacos que sean mejor tolerados y que se ajusten más a las preferencias de los pacientes, aunque de los pacientes estudiados el $52 \%$ de no tuvo preferencias por ningún fármaco del MOM.

También es conocido, que un elevado porcentaje de pacientes abandona el tratamiento para evitar la sintomatología que les producen las reacciones adversas, independientemente de los beneficios clínicos que éstos le puedan ofrecer. De hecho, los fármacos que inducen con frecuencia sintomatología digestiva pueden suponer un mayor riesgo de no cumplimiento, sin embargo solo un $10 \%$ de los pacientes en diálisis refirió intolerancia gástrica a estos fármacos.

Entre las limitaciones de este estudio, tenemos que no se analizó la prevalencia de ansiedad y depresión en estos pacientes en hemodiálisis, que se ha descrito como eleva$\mathrm{da}^{3}$. La identificación e intervención psicológica en los pacientes con estas patologías podría ser considerada como parte del cuidado en estos pacientes y posiblemente podría aumentar el grado de cumplimiento. Aspectos como éste se deberían trabajar en un futuro para minimizar la falta de cumplimiento terapéutico en esta población.

Finalmente, para prevenir potenciales pacientes no adherentes, primero debemos identificarles, para posteriormente poder actuar. Esto nos dará la oportunidad de intervenir para intentar modificar su comportamiento, además de facilitarnos la adopción enfoques terapéuticos, evitando aumentos innecesarios de las dosis de los fármacos prescritos (debido en teoría a la falta de la respuesta al tratamiento), cuando la realidad subyacente es la falta de cumplimiento.

\section{Conclusiones}

Como conclusiones de nuestro estudio, el $37,7 \%$ de los pacientes en programa de hemodiálisis de nuestro centro, fue no adherente al tratamiento médico prescrito, según las respuestas obtenidas en el cuestionario SMAQ, y que el grado de conocimiento, información y/o compresión sobre el tratamiento médico-dietético prescrito a los pacientes son factores muy importantes para mejorar el grado de cumplimiento, por lo que la implicación activa el personal de enfermería constituye una herramienta clave para conseguir el objetivo terapéutico. 
Recibido: 20 Febrero 2013

Revisado: 25 Febrero 2013

Modificado: 1 Marzo 2013

Aceptado: 2 Marzo 2013

\section{Bibliografía}

1. Morisky DE, Green LW, Levine DM. Concurrent and predictive validity of a self- reported measure of medication adherence. Med Care 1986;24(1):67-74.

2. Knobel H, Alonso J, Casado JL, Collazos J, González J, Ruiz I, et al. GEEMA Study Group. Validation of a simplified medication adherence questionnaire in a large cohort of HIV-infected patients: the GEEMA study. AIDS 2002;16:605-13.

3. Arenas MD, Malek T, Álvarez-Ude F, Gil M.T, et al. Captores del fósforo: preferencias de los pacientes en hemodiálisis y su repercusión sobre el cumplimiento del tratamiento y el control del fósforo.Nefrología 2010; 30(5):522-30.

4. F.J. Ortega Suárez, J. Sánchez Plumed, M.A. Pérez Valentín, P. Pereira Palomo, M.A. Muñoz Cepada, D. Lorenzo Aguiar, Grupo de Estudio Vatren. Validación del cuestionario simplificado de adherencia a la medicación (SMAQ) en pacientes con transplante renal en terapia con tacrolimus. Nefrología 2011;31(6):690-6.

5. Martín Alfonso L. Acerca del concepto de adherencia terapéutica. Rev. Cubana Salud Pública, 2004; 30(4):350-2.
6. Ochando G, Royuela G, Hernández $M$, Lorenzo M, Paniagua F. Rev. Soc. Esp. Enferm. Nefrol. 2008; 11(4): 271/276.

7. Karamanidou C, Clatworthy J, Weinman J, Horne R.A systematic review of the prevalence and determinants of no adherence to phosphate binding medication in patients with end-stage renal disease. BMC Nephrology 2008;9:2 doi:10.1186/1471-2369-9-2.

8. Kammerer J, Garry G, Hartingan M, Carter B, Erlich L. Adherence in patients on dialysis: strategies for success. Nephrology Nursing Journal 2007;34 (5).

9. Vila P, García P N, Gómez R, García P R, Tejuca M M, Tejuca M A. Cumplimentaciónterapeútica de pacientes de dialysis. En: Libro de comunicaciones del XXXII Congreso de la Sociedad Española de Enfermería Nefrológica; Cádiz 3-6 octubre 2007. p. 173-177.

10. Ami J. Claxton, MS, PhD, Joyce Cramer, BS, Courtney Pierce. A systematic review of the associations between dose regimens and medication compliance. Clinical Therapeutics 2001; 23(8).

11. Horne R, et al. (2004). Doubts about necessity and concerns about adverse events: identifying the types of beliefs that are associated with non-adherence to HAART.

12. Sánchez V, Lorenzo S. Hemodiálisis: ¿Cuánto sabemos de los fármacos relacionados con el metabolismo mineral?.Rev Soc Esp Nefrol 2011; 14 (1): 23/29. 